\title{
$q$-EXTENSION OF THE $p$-ADIC GAMMA FUNCTION \\ BY
}

NEAL KOBLITZ

\begin{abstract}
. $p$-adic functions depending on a parameter $q, 0<|q-1|_{p}<1$, are defined which extend $Y$. Morita's $p$-adic gamma function and the derivative of $J$. Diamond's $p$-adic log-gamma function in the same way as the classical $q$-gamma function $\Gamma_{q}(x)$ extends $\Gamma(x)$. Properties of these functions which are analogous to the basic identities satisfied by $\Gamma_{q}(x)$ are developed.
\end{abstract}

1. Introduction. A generalized gamma function $\Gamma_{q}(x)$, depending on a parameter $0<q<1$, was introduced and studied by F. H. Jackson, R. Askey, G. E. Andrews and others. Defined as

$$
\Gamma_{q}(x)=(1-q)^{1-x} \frac{(1-q)\left(1-q^{2}\right)\left(1-q^{3}\right) \cdots}{\left(1-q^{x}\right)\left(1-q^{x+1}\right)\left(1-q^{x+2}\right) \cdots},
$$

it satisfies relations which generalize the well-known identities for the gamma function, and in the limit as $q \rightarrow 1^{-}$it becomes $\Gamma(x)$.

The purpose of this article is to construct and study the properties of a natural $q$-extension $\Gamma_{p, q}$ of Morita's $p$-adic gamma function $\Gamma_{p}$ [13] and a $q$-extension $\psi_{p, q}$ of the derivative of Diamond's $p$-adic log-gamma function $G_{p}$ [4]. Recall that $\Gamma_{p}$ is a function from the $p$-adic integers $\mathbf{Z}_{p}$ to the $p$-adic units $\mathbf{Z}_{p}^{*}$ defined by

$$
\Gamma_{p}(x)=\lim _{n \rightarrow x}(-1)^{n} \prod_{j<n}^{\prime} j,
$$

where $n$ runs over positive integers and $\Pi^{\prime}$ means that indices $j$ divisible by $p$ are omitted. On the other hand, $G_{p}$ is a function on the complement of $\mathbf{Z}_{p}$ in $\Omega_{p}$ (where $\Omega_{p}$ is the $p$-adic completion of the algebraic closure of the field $\mathbf{Q}_{p}$ of $p$-adic numbers, with norm ||$_{p}$ for which $\left.|p|_{p}=p^{-1}\right)$. It is defined as

$$
G_{p}(x)=\lim _{N \rightarrow \infty} p^{-N} \sum_{0<j<p^{N}}(x+j)\left(\log _{p}(x+j)-1\right),
$$

where $\log _{p}$ is the Iwasawa $p$-adic logarithm [7]. Although $G_{p}$ is not $\log _{p} \Gamma_{p}$, it has the following two connections with $\log _{p} \Gamma_{p}$ (see [4] and [6]). (1) If we let $G_{p}^{*}(x)=$ $G_{p}(x)-G_{p}(x / p)$, which is defined on the complement of $\mathbf{Z}_{p}^{*}$ by the same formula as $G_{p}$ with $\Sigma$ replaced by $\Sigma^{\prime}$, then $G_{p}^{*}(x)=\log _{p} \Gamma_{p}(x)$ for $x \in p Z_{p}$. (2) $\log _{p} \Gamma_{p}(x)$ $=\Sigma_{0<j<p, x+j \notin p \mathbf{Z}_{p}} G_{p}((x+j) / p)$ for $x \in \mathbf{Z}_{p}$.

For simplicity suppose $p>2$.

Received by the editors October 2, 1979 and, in revised form, November 14, 1979.

AMS (MOS) subject classifications (1970). Primary 12B40; Secondary 33A15.

Key words and phrases. Gamma function, p-adic functions, $q$-extension, Euler constants.

() 1980 American Mathematical Society 0002-9947/80/0000-0357/\$03.25 


\section{2. $q$-extension of $\Gamma_{p}$.}

TheOREM 1. Let $t \in \Omega_{p},|t|_{p}<1, t \neq 0$. Set $q=1+t$, and for any $n$ define

$$
\Gamma_{p, q}(n)=(-1)^{n} \prod_{j<n}^{\prime} \frac{1-q^{j}}{1-q} .
$$

Then $\Gamma_{p, q}$ extends to a continuous function on $\mathbf{Z}_{p}$, and $\lim _{q \rightarrow 1} \Gamma_{p, q}=\Gamma_{p}$.

Proof. We first prove two general lemmas.

Lemma 1. Let $P_{1}(X), \ldots, P_{h}(X) \in \mathbf{Q}[X]$. Then there exists $Q(X) \in \mathbf{Q}[X]$ such that for all $n$

$$
\sum_{i_{1}<i_{2}<\cdots<i_{n}<n} P_{1}\left(i_{1}\right) \cdots P_{h}\left(i_{h}\right)=Q(n) .
$$

Proof of Lemma. Use induction on $h$. For $h=1$ the lemma follows because $\sum_{i_{1}=1}^{n-1} i_{1}^{l}$ is a polynomial in $n$ for any $l$. Suppose the lemma holds for $h-1$. We have

$$
\begin{aligned}
\sum_{i_{1}<i_{2}<\cdots<i_{h}<n} P_{1}\left(i_{1}\right) \cdots P_{h}\left(i_{h}\right) & =\sum_{i_{2}<\cdots<i_{h}<n}\left(\sum_{i_{1}<i_{2}} P_{1}\left(i_{1}\right)\right) P_{2}\left(i_{2}\right) \cdots P_{h}\left(i_{h}\right) \\
& =\sum_{i_{2}<\cdots<i_{h}<n}\left(Q_{1}\left(i_{2}\right) P_{2}\left(i_{2}\right)\right) P_{3}\left(i_{3}\right) \cdots P_{h}\left(i_{h}\right)
\end{aligned}
$$

by the lemma for $h=1$. But this is of the form $Q(n)$ by the lemma for $h-1$ applied to the polynomials $Q_{1} P_{2}, P_{3}, \ldots, P_{h}$.

Lemma 2. Let $P_{k}(X) \in \mathrm{Q}[X], k=1,2, \ldots ; A_{j}(t)=1+\sum_{k=1}^{\infty} P_{k}(j) t^{k} \in \mathrm{Q}[[t]]$, $j=1,2, \ldots$ Then there exist $Q_{k}(X) \in \mathbf{Q}[X]$ such that for all $n$,

$$
\prod_{j<n} A_{j}(t)=1+\sum_{k=1}^{\infty} Q_{k}(n) t^{k}
$$

Proof of Lemma. For each $k$ let $s=\left\{s_{1}, \ldots, s_{h}\right\}$ run through the set $S$ of partitions of ordered positive integers $s_{i}$ whose sum is $k$. The coefficient of $t^{k}$ in $\Pi_{j<n} A_{j}(t)$ is clearly

$$
\sum_{s \in S} \sum_{i_{1}<i_{2}<\cdots<i_{h}<n} P_{s_{1}}\left(i_{1}\right) \cdots P_{s_{h}}\left(i_{h}\right) .
$$

Since the first sum is finite, this is a polynomial in $n$ by Lemma 1.

Proof of Theorem 1. Let $P_{k}(X)=(X-1)(X-2) \cdots(X-k) /(k+1)$ !. Then

$$
\frac{1-q^{j}}{1-q}=\frac{(1+t)^{j}-1}{t}=j\left(1+\sum_{k=1}^{\infty} P_{k}(j) t^{k}\right)
$$

Further let $\tilde{n}=[(n-1) / p]+1$, and let $\tilde{P}_{k}(X)=P_{k}(p X)$. Finally, let

$$
A_{j}(t)=1+\sum_{k=1}^{\infty} P_{k}(j) t^{k}, \quad \tilde{A}_{j}(t)=1+\sum_{k=1}^{\infty} \tilde{P}_{k}(j) t^{k}
$$


Then

$$
\Gamma_{p, q}(n)=\Gamma_{p}(n) \frac{\Pi_{j<n} A_{j}(t)}{\prod_{j<\tilde{n}} \tilde{A}_{j}(t)}
$$

By Lemma 2, the quotient can be written $\left(1+\sum Q_{k}(n) t^{k}\right) /\left(1+\sum \tilde{Q}_{k}(\tilde{n}) t^{k}\right)$.

Note that $\Gamma_{p, q}(n)=(-1)^{n} \Pi_{j<n, p \nmid j}\left(1+(1+t)+\cdots+(1+t)^{j-1}\right) \in Z[t]$, and hence $\Gamma_{p, q}(n) / \Gamma_{p}(n)=1+\sum_{k=1}^{\infty} R_{k}(n) t^{k} \in \mathbf{Z}_{p}[[t]]$ for each $n$ (where the $R_{k}$ are some functions of $n$ ), i.e., $\left|R_{k}(n)\right|_{p} \leqslant 1$ for all $k$, $n$. Since each $R_{k}$ is a finite expression in $Q_{k_{1}}$ and $\tilde{Q}_{k_{2}}, k_{1}, k_{2} \leqslant k$, and since each $\tilde{Q}_{k_{2}}$ depends continuously on $\tilde{n}$ and hence on $n$, and $Q_{k_{1}}$ is just a polynomial in $n$, it follows that $R_{k}$ is a bounded (by 1) continuous function of $n$. Since $|t|_{p}<1$, the theorem follows. Q.E.D.

THEOREM 2.

(1)

$$
\Gamma_{p, q}(x+1) / \Gamma_{p, q}(x)=\left\{\begin{array}{l}
-\left(1-q^{x}\right) /(1-q) \\
-1 \text { if } x \in p \mathbf{Z}_{p}
\end{array} \text { if } x \in \mathbf{Z}_{p}^{*}\right.
$$

$$
\begin{aligned}
\Gamma_{p, q}(x) \Gamma_{p, q^{m}}\left(\frac{1}{m}\right) \Gamma_{p, q^{m}}\left(\frac{2}{m}\right) \cdots \Gamma_{p, q^{m}}\left(\frac{m-1}{m}\right) \\
\quad=\Gamma_{p, q^{m}}\left(\frac{x}{m}\right) \Gamma_{p, q^{m}}\left(\frac{x+1}{m}\right) \cdots \Gamma_{p, q^{m}}\left(\frac{x+m-1}{m}\right)\left(\frac{1-q^{m}}{1-q}\right)^{x-\bar{x}}
\end{aligned}
$$

for any $x \in \mathbf{Z}_{p}$ and any positive integer $m$ prime to $p$, where $\sim$ is the unique continuous extension to $\mathbf{Z}_{p}$ of the map $n \mapsto \tilde{n}=[(n-1) / p]+1$ used in the proof of Theorem 1. (If $x=a_{0}+a_{1} p+a_{2} p^{2}+\cdots$, then $\tilde{x}=\left(a_{1}+1\right)+a_{2} p+a_{3} p^{2}$ $+\cdots$ if $a_{0} \neq 0$ and $a_{1}+a_{2} p+a_{3} p^{2}+\cdots$ if $a_{0}=0$. Note that $\left(1-q^{m}\right) /(1-q)$ is a p-adic unit, and the exponent can be written $a_{0}-p+(p-1) \tilde{x}$ if $a_{0} \neq 0$ and $(p-1) \tilde{x}$ if $a_{0}=0$.)

$$
\Gamma_{p, q}(x) \Gamma_{p, 1 / q}(1-x)=-(-q)^{\bar{x}-x}
$$

Proof. Since both sides of (1), (2) and (3) are continuous in $x$, it suffices to prove them for $x=n$. In that case (1) follows immediately from the definition.

(2) For fixed $m$ let $A_{n}$ denote the left side of (3) for $x=n$, and let $B_{n}$ denote the right side. We prove that $A_{n}=B_{n}$ by induction on $n$. Trivially $A_{1}=B_{1}$. Suppose $A_{n}=B_{n}$. We have

$$
\frac{A_{n+1}}{A_{n}}=\frac{\Gamma_{p, q}(n+1)}{\Gamma_{p, q}(n)}=\left\{\begin{array}{l}
-\left(1-q^{n}\right) /(1-q) \text { if } p \nmid n, \\
-1 \text { if } p \mid n,
\end{array}\right.
$$

and

$$
\begin{aligned}
\frac{B_{n+1}}{B_{n}} & =\frac{\Gamma_{p, q^{m}}(n / m+1)}{\Gamma_{p, q^{m}}(n / m)}\left(\frac{1-q^{m}}{1-q}\right)^{1+\tilde{n}-(\widetilde{n+1})} \\
& = \begin{cases}\left(-\left(1-q^{n}\right) /\left(1-q^{m}\right)\right) \cdot\left(\left(1-q^{m}\right) /(1-q)\right) \text { if } p \nmid n, \\
-1 \cdot 1 & \text { if } p \mid n .\end{cases}
\end{aligned}
$$


Hence $A_{n+1} / A_{n}=B_{n+1} / B_{n}$, and so $A_{n+1}=B_{n+1}$. This completes the induction.

(3) is easily proved by induction in the same way as (2). Q.E.D.

Remarks. 1. Comparing parts (2) and (3) of Theorem 2 gives support for a comment once made by B. H. Gross that the Euler reflection formula for the gamma function should be thought of as the (-1)-case of the multiplication formula. The right side of (3) can be written as

$$
\Gamma_{p, q}(1)\left(\frac{1-q^{-1}}{1-q}\right)^{x-\tilde{x}}
$$

(recall $\left.\Gamma_{p, q}(1)=-1\right)$. This similarity between the multiplication and the reflection formulas for $\Gamma_{p}$ was not clear until we looked at its $q$-extension $\Gamma_{p, q}$.

2 . In the classical case the type of argument in Theorem 2 above will quickly reveal that $\Gamma_{q}(x) \Gamma_{1 / q}(1-x)(-q)^{x}$ is periodic of period 1 (where we use D. Moak's $\Gamma_{q}$ for $q>1[11]$ to define $\Gamma_{1 / q}(1-x)$ ). But this periodic function is not a constant (as any continuous function on $\mathbf{Z}_{p}$ with period 1 must be); it turns out to equal a constant divided by the theta-function

$$
\sum_{n=-\infty}^{\infty}(-1)^{-(n+x)} q^{(n+x-1 / 2)^{2} / 2}
$$

\section{Table of properties.}

\section{Classical Case}

gamma function

its $q$-extension $(0<q<1)$

(1) $\Gamma(n+1)=n$ !

$\Gamma_{q}(n+1)=\prod_{j=1}^{n}\left(1+q+q^{2}+\cdots+q^{j-1}\right)$

(2) $\frac{\Gamma(x+1)}{\Gamma(x)}=x, \quad \Gamma(1)=1 \quad \frac{\Gamma_{q}(x+1)}{\Gamma_{q}(x)}=\frac{\left(1-q^{x}\right)}{(1-q)}, \quad \Gamma(1)=1$.

$\Gamma$ and $\Gamma_{q}$ are uniquely characterized by (2) together with convexity of their logarithm.

(3) $\Gamma(x) \Gamma\left(\frac{1}{m}\right) \cdots \Gamma\left(\frac{m-1}{m}\right)$

$$
\begin{aligned}
& \Gamma_{q}(x) \Gamma_{q^{m}}\left(\frac{1}{m}\right) \cdots \Gamma_{q^{m}}\left(\frac{m-1}{m}\right) \\
& =\Gamma_{q^{m}}\left(\frac{x}{m}\right) \Gamma_{q^{m}}\left(\frac{x+1}{m}\right) \cdots \\
& \Gamma_{q^{m}}\left(\frac{x+m-1}{m}\right)\left(\frac{\left(1-q^{m}\right)}{(1-q)}\right)^{x-1}
\end{aligned}
$$$$
=\Gamma\left(\frac{x}{m}\right) \Gamma\left(\frac{x+1}{m}\right) \cdots
$$$$
\Gamma\left(\frac{x+m-1}{m}\right) m^{x-1}
$$

for any positive integer $m$

for any positive integer $m$

(4) $\Gamma(x) \cdot \Gamma(1-x)=\frac{\pi}{\sin (\pi x)}$

$$
\begin{aligned}
& \Gamma_{q}(x) \Gamma_{q}(1-x) \\
& =\sum_{n=-\infty}^{\infty} \frac{(1-q)(-1)^{n} q^{n(n+1) / 2}}{\left(1-q^{n+x}\right)}
\end{aligned}
$$

(5) $\operatorname{dlog} \Gamma(1)=-\gamma$

$\operatorname{dlog} \Gamma_{q}(1)=-\gamma_{q}$. 


\section{$p$-adic Case}

gamma function

(1) $\Gamma_{p}(n+1)=(-1)^{n+1} \prod_{j=1}^{n} j$ its $q$-extension $\left(0<|q-1|_{p}<1\right)$

$$
\begin{aligned}
& \Gamma_{p, q}(n+1)=(-1)^{n+1} \prod_{j=1}^{n} \\
& \cdot\left(1+q+q^{2}+\cdots+q^{j-1}\right)
\end{aligned}
$$

(2) $\frac{\Gamma_{p}(x+1)}{\Gamma_{p}(x)}=\left\{\begin{array}{l}-x \text { if } x \in \mathbf{Z}_{p}^{*}, \\ -1 \text { if } x \in p \mathbf{Z}_{p}\end{array}\right.$

$$
\Gamma_{p}(1)=-1
$$

$$
\begin{aligned}
& \frac{\Gamma_{p, q}(x+1)}{\Gamma_{p, q}(x)}=\left\{\begin{array}{l}
-\frac{\left(1-q^{x}\right)}{(1-q)} \text { if } x \in \mathbf{Z}_{p}^{*}, \\
-1 \text { if } x \in p \mathbf{Z}_{p},
\end{array}\right. \\
& \Gamma_{p, q}(1)=-1 .
\end{aligned}
$$

$\Gamma_{p}$ and $\Gamma_{p, q}$ are uniquely characterized by (2) together with continuity.

(3) $\Gamma_{p}(x) \Gamma_{p}\left(\frac{1}{m}\right) \cdots \Gamma_{p}\left(\frac{m-1}{m}\right)$

$=\Gamma_{p}\left(\frac{x}{m}\right) \Gamma_{p}\left(\frac{x+1}{m}\right) \cdots$

$\Gamma_{p}\left(\frac{x+m-1}{m}\right) m^{x-\tilde{x}}$

for any positive integer $m$ prime to $p$

(4) $\Gamma_{p}(x) \cdot \Gamma_{p}(1-x)=(-1)^{x-\tilde{x}} \Gamma_{p}(1)$

(5) $\operatorname{dlog} \Gamma_{p}(0)=-(1-1 / p) \gamma_{p}$

$$
\begin{aligned}
& \Gamma_{p, q}(x) \Gamma_{p, q^{m}}\left(\frac{1}{m}\right) \cdots \Gamma_{p, q^{m}}\left(\frac{m-1}{m}\right) \\
& =\Gamma_{p, q^{m}}\left(\frac{x}{m}\right) \Gamma_{p, q^{m}}\left(\frac{x+1}{m}\right) \\
& \cdots \Gamma_{p, q^{m}}\left(\frac{x+m-1}{m}\right)\left(\frac{1-q^{m}}{1-q}\right)^{x-\tilde{x}}
\end{aligned}
$$

for any positive integer $m$ prime to $p$

$$
\begin{aligned}
& \Gamma_{p, q}(x) \cdot \Gamma_{p, 1 / q}(1-x) \\
& =(-q)^{\tilde{x}-x} \Gamma_{p, q}(1)
\end{aligned}
$$

$\operatorname{dlog} \Gamma_{p, q}(0)=-(1-1 / p) \gamma_{p, q}$

(see below)

Finally, $\Gamma_{q} \rightarrow \Gamma$ as $q \rightarrow 1^{-}$, and $\Gamma_{p, q} \rightarrow \Gamma_{p}$ as $q \rightarrow 1$.

4. $q$-extension of $G_{p}^{\prime}$. For $x \in \Omega_{p}$ let $d(x)=\min _{z \in Z_{p}}|x-z|_{p}$ be the distance from $x$ to $\mathbf{Z}_{p}$. For $q=1+t,|t|_{p}<1$, let $\varepsilon_{q}=1 /\left(\left|\log _{p} q\right|_{p} \cdot p^{1 /(p-1)}\right)$, and let $D_{q}=\{x \in$ $\left.\Omega_{p} \mid 0<d(x)<\varepsilon_{q}\right\}$. Thus, if ord $t>1 /(p-1)$, we have $\varepsilon_{q}=1 /\left(|t|_{p} \cdot p^{1 /(p-1)}\right)>1$ and $D_{q}=\left\{x \in \Omega_{p}-\left.Z_{p}|| x\right|_{p}<\varepsilon_{q}\right\}$; as $q \rightarrow 1, D_{q}$ expands to all of $\Omega_{p}-\mathbf{Z}_{p}$.

If $x \in D_{q}$, say $|x-z|_{p}<\varepsilon_{q}$ with $z \in \mathbf{Z}_{p}$, then $q^{x}=q^{z} e^{(x-z) \log _{p} q}$, and so $q^{x}$ is a locally analytic function on $D_{q}$. Since also $q^{x} \neq 1$ here, it follows that $\log _{p}\left(1-q^{x}\right)$ is locally analytic on $D_{q}$. We can then define

$$
\begin{aligned}
\psi_{p, q}(x) & =\lim _{N \rightarrow \infty} p^{-N} \sum_{0<j<p^{N}} \log _{p} \frac{1-q^{x+j}}{1-q} \\
& =-\log _{p}(1-q)+\lim _{N \rightarrow \infty} p^{-N} \sum_{0<j<p^{N}} \log _{p}\left(1-q^{x+j}\right)
\end{aligned}
$$


which exists and is locally analytic on $D_{q}$ by Theorem 2 of [4]. Clearly $\psi_{p, q} \rightarrow G_{p}^{\prime}$ as $q \rightarrow 1$.

We can define $\psi_{p, q}^{*}(x)$-a $q$-extension of $G_{p}^{* \prime}$-by replacing $d(x)$ by $d^{*}(x)=$ $\min _{z \in \mathbf{Z}_{p}^{*}}|x-z|_{p}, D_{q}$ by $D_{q}^{*}=\left\{x \in \Omega_{p} \mid 0<d^{*}(x)<\varepsilon_{q}\right\}$, and $\Sigma$ by $\Sigma^{\prime}$ in the definition of $\psi_{p, q}$. Then

$$
\psi_{p, q}^{*}(x)=\psi_{p, q}(x)-\frac{1}{p} \psi_{p, q^{p}}\left(\frac{x}{p}\right)-\frac{1}{p} \log _{p} \frac{1-q^{p}}{1-q} \quad \text { for } x \in D_{q}
$$

We first show the relationship between $\psi_{p, q}(x)$ and $d \log _{p} \Gamma_{p, q}(x) / d x$.

THEOREM 3.

(1)

$$
\psi_{p, q}^{*}(x)=\frac{d}{d x} \log _{p} \Gamma_{p, q}(x) \quad \text { for } x \in p \mathbf{Z}_{p}
$$

(2)

$$
\frac{d}{d x} \log _{p} \Gamma_{p, q}(x)=\frac{1}{p} \sum_{\substack{0<j<p \\ x+j \notin p \mathbf{Z}_{p}}} \psi_{p, q^{p}}\left(\frac{x+j}{p}\right)+\left(1-\frac{1}{p}\right) \log _{p} \frac{1-q^{p}}{1-q} \text { for } x \in \mathbf{Z}_{p}
$$

Note that if $\operatorname{ord}_{p}(q-1)>1 /(p-1)$, then $\varepsilon_{q}>1$ and $\varepsilon_{q^{p}}>p$, so that $x \in D_{q}^{*}$ and $(x+j) / p \in D_{q^{p}}$. But even if $\operatorname{ord}_{p}(q-1)<1 /(p-1)$, in which case we may have $x \notin D_{q}^{*}$ and $(x+j) / p \notin D_{q^{p}}$, still $\psi_{p, q}^{*}(x)$ in (1) and $\psi_{p, q^{p}}((x+j) / p)$ in (2) are well defined because $x \in p \mathbf{Z}_{p}$ and $x \in \mathbf{Z}_{p}$, respectively.

Proof. (1) By the definition of $\Gamma_{p, q}$, for $x \in p Z_{p}$ we have

$$
p^{-N}\left(\log _{p} \Gamma_{p, q}\left(x+p^{N}\right)-\log _{p} \Gamma_{p, q}(x)\right)=p^{-N} \sum_{0<j<p^{N}}^{\prime} \log _{p} \frac{1-q^{x+j}}{1-q},
$$

and taking the limit as $N \rightarrow \infty$ gives (1).

(2) The sum on the right in (2) is equal to

$$
\begin{aligned}
\lim _{N \rightarrow \infty} p^{-N-1} \sum_{\substack{0<j<p^{N} \\
0<k<p \\
x+k \notin p \mathbf{Z}_{p}}} \log _{p} \frac{1-q^{p((x+k) / p+j)}}{1-q^{p}} \\
=-\left(1-\frac{1}{p}\right) \log _{p} \frac{1-q^{p}}{1-q}+\lim _{N \rightarrow \infty} p^{-N} \sum_{\substack{0<j<p^{N} \\
x+j \notin p \mathbf{Z}_{p}}} \log _{p} \frac{1-q^{x+j}}{1-q} \\
=-\left(1-\frac{1}{p}\right) \log _{p} \frac{1-q^{p}}{1-q}+\lim _{N \rightarrow \infty} p^{-N}\left(\log _{p} \Gamma_{p, q}\left(x+p^{N}\right)-\log _{p} \Gamma_{p, q}(x)\right) \\
=-\left(1-\frac{1}{p}\right) \log _{p} \frac{1-q^{p}}{1-q}+\frac{d}{d x} \log _{p} \Gamma_{p, q}(x) . \text { Q.E.D. }
\end{aligned}
$$

The next theorem, giving identities for $\psi_{p, q}$, should be compared with the table in §3. 
THEOREM 4. For $x \in D_{q}$,

(1)

$$
\psi_{p, q}(x+1)-\psi_{p, q}(x)=-\frac{q^{x} \log _{p} q}{1-q^{x}}=\frac{d}{d x} \log _{p} \frac{1-q^{x}}{1-q},
$$

(2)

$$
\psi_{p, q}(x)-\frac{1}{m} \sum_{h=0}^{m-1} \psi_{p, q^{m}}\left(\frac{x+h}{m}\right)=\log _{p} \frac{1-q^{m}}{1-q}=\frac{d}{d x} \log _{p}\left(\frac{1-q^{m}}{1-q}\right)^{x-1}
$$

for any positive integer $m$ (not necessarily prime to $p$ ),

(3)

$$
\psi_{p, q}(x)-\psi_{p, 1 / q}(1-x)=-\log _{p} q=\frac{d}{d x} \log _{p}(-q)^{-x} .
$$

Proof. (1) This follows by Theorem 4 of [4]:

$$
\begin{aligned}
\psi_{p, q}(x+1)-\psi_{p, q}(x) & =\lim _{N \rightarrow \infty} p^{-N}\left(\log _{p} \frac{1-q^{x+p^{N}}}{1-q}-\log _{p} \frac{1-q^{x}}{1-q}\right) \\
& =\frac{d}{d x} \log _{p} \frac{1-q^{x}}{1-q} .
\end{aligned}
$$

(2) By Theorem 1(ii) of [4], $\psi_{p, q}(x)$ can also be written (for any $m$ ) as

$$
\lim _{N \rightarrow \infty} \frac{1}{m p^{N}} \sum_{0<j<m p^{N}} \log _{p} \frac{1-q^{x+j}}{1-q} .
$$

Then the left side in (2) equals

$$
\begin{aligned}
& \lim _{N \rightarrow \infty}\left(\frac{1}{m p^{N}} \sum_{0<j<m p^{N}} \log _{p} \frac{1-q^{x+j}}{1-q}\right.\left.-\frac{1}{m p^{N}} \sum_{0<j<p^{N}} \sum_{0<h<m} \log _{p} \frac{1-q^{m((x+h) / m+j)}}{1-q^{m}}\right) \\
&=\lim _{N \rightarrow \infty} \frac{1}{m p^{N}} \sum_{0<j<m p^{N}}\left(\log _{p} \frac{1-q^{x+j}}{1-q}-\log _{p} \frac{1-q^{x+j}}{1-q^{m}}\right)=\log _{p} \frac{1-q^{m}}{1-q} .
\end{aligned}
$$

(3)

$$
\begin{aligned}
\psi_{p, q}(x) & -\psi_{p, 1 / q}(1-x) \\
& =\lim _{N \rightarrow \infty} p^{-N} \sum_{0<j<p^{N}}\left(\log _{p} \frac{1-q^{x+j}}{1-q}-\log _{p} \frac{1-q^{-\left(1-x+p^{N}-1-j\right)}}{1-q^{-1}}\right) \\
& =-\log _{p} q+\lim _{N \rightarrow \infty} p^{-N} \sum_{0<j<p^{N}} \log _{p} \frac{1-q^{x+j}}{1-q^{x+j-p^{N}}} .
\end{aligned}
$$


Since

$$
\begin{aligned}
\log _{p} \frac{1-q^{x+j}}{1-q^{x+j-p^{N}}} & =-\log _{p}\left(1-\frac{q^{x+j}}{1-q^{x+j}}\left(q^{-p^{N}}-1\right)\right) \\
& =\sum_{k=1}^{\infty} \frac{1}{k} \frac{q^{(x+j) k}}{\left(1-q^{x+j}\right)^{k}}\left(q^{-p^{N}}-1\right)^{k}
\end{aligned}
$$

and

$$
q^{-p^{N}}-1=(1+t)^{-p^{N}}-1=-p^{N} t+p^{N}\left(p^{N}+1\right) t^{2} / 2-\cdots,
$$

it quickly follows that the last limit is zero. Q.E.D.

Finally, we give $q$-extensions of Diamond's $p$-adic Euler constants [4]. If $r, f \in$ $\mathbf{Z}, f \geqslant 1$, and if $\operatorname{ord}_{p}(r / f)<0$, then define

$$
\gamma_{p, q}(r, f)=-\lim _{N \rightarrow \infty} \frac{1}{f p^{N}} \sum_{\substack{0<j<f^{N} \\ j \equiv r(\bmod f)}} \log _{p} \frac{1-q^{j}}{1-q}
$$

Also set

$$
\gamma_{p, q}=\frac{p}{p-1} \sum_{j=1}^{p-1} \gamma_{p, q}(j, p)=-\frac{p}{p-1} \lim _{N \rightarrow \infty} p^{-N} \sum_{0<j<p^{N}}^{\prime} \log _{p} \frac{1-q^{j}}{1-q}
$$

Then it is easy to prove the following two theorems, which generalize Theorem 14 in [4].

THEOREM 5. (1) If $d \mid(r, f)$, then

$$
f \gamma_{p, q}(r, f)=(f / d) \gamma_{p, q^{d}}(r / d, f / d)-\log _{p} \frac{1-q^{d}}{1-q}
$$

(2) $\gamma_{p, q}(r, f)=\gamma_{p, 1 / q}(f-r, f)+(1 / f) \log _{p} q$.

(3) If $b$ is a positive integer, then $\gamma_{p, q}(r, f)=\sum_{j=0}^{b-1} \gamma_{p, q}(r+j f$, bf).

THEOREM 6. (1) If $\operatorname{ord}_{p}(r / f)<0$ and $0<r<f$, then

$$
\psi_{p, q^{f}}(r / f)=-\log _{p} \frac{1-q^{f}}{1-q}-f \gamma_{p, q}(r, f) .
$$

(2) $\psi_{p, q}^{*}(0)=\Gamma_{p, q}^{\prime}(0)=-(1-1 / p) \gamma_{p, q}$.

REMARKS. 1. In [4] Diamond denotes a limit of the form $\lim _{N \rightarrow \infty} p^{-N} \Sigma_{0 \leqslant j<p^{N}} f(j, x)$ by $\int f(u, x) d u$. This is the Riemann sum definition of the integral over $\mathbf{Z}_{p}$ with respect to the Haar distribution $\mu_{\mathrm{Haar}}\left(j+p^{N} \mathbf{Z}_{p}\right)=p^{-N}$ if one chooses $j$ as the representative "point" in the "interval" $j+p^{N} \mathbf{Z}_{p}$; since $\mu_{\text {Haar }}$ is not bounded, the limit of the Riemann sums does not exist independently of the choice of representative. The relationship between $\mu_{\mathrm{Haar}}$ and the $\mu_{z}$ in [9] given by $\mu_{z}\left(j+p^{N} \mathbf{Z}_{p}\right)=z^{j} /\left(1-z^{p^{N}}\right)$ is as follows. If we choose $z=q$ with $|q-1|_{p}<1$ (but $q$ not a $p$ th-power root of 1 ), then $\mu_{q}$ is an unbounded distribution, and $d \mu_{\mathrm{Haar}}=-q^{-u} \log _{p} q d \mu_{q}=(d / d u)\left(q^{-u}\right) d \mu_{q}$ in the sense that 


$$
\begin{aligned}
\lim _{N \rightarrow \infty} \sum_{0<j<p^{N}} f(j, x) \mu_{\text {Haar }}\left(j+p^{N} \mathbf{Z}_{p}\right) \\
\quad=\lim _{N \rightarrow \infty} \sum_{0<j<p^{N}} f(j, x)\left(-q^{-j} \log _{p} q\right) \mu_{q}\left(j+p^{N} \mathbf{Z}_{p}\right) .
\end{aligned}
$$

2. If we wanted to construct a $q$-extension $G_{p, q}$ of $G_{p}$ having the form $G_{p, q}(x)=$ $\lim _{N \rightarrow \infty} p^{-N} \Sigma_{0 \leqslant j<p^{N}} f(x+j)$, we would have to find an $f$ whose derivative is $\log _{p}\left(\left(1-q^{x}\right) /(1-q)\right)$. It is not clear what a natural choice for such an $f$ might be. However, one could use the technique in [9] to define a "twisted" $G_{p, q}$ by

$$
G_{p, q, \xi}(x)=-\int_{\mathbf{Z}_{p}} \log _{p} \frac{1-q^{x+u}}{1-q} d \mu_{\xi}(u) \text { for } x \in D_{q},
$$

where $\xi^{r}=1, \xi \neq 1, p \nmid r$, and $\mu_{\xi}\left(j+p^{N} \mathbf{Z}_{p}\right)=\xi^{j} /\left(1-\xi^{p^{N}}\right)$. This function satisfies

(1)

$$
\xi G_{p, q, \xi}(x+1)-G_{p, q, \xi}(x)=\log _{p} \frac{1-q^{x}}{1-q}
$$

(2)

$$
G_{p, q, \xi}(x)-\sum_{h=0}^{m-1} \xi^{h} G_{p, q^{m}, \xi^{m}}\left(\frac{x+h}{m}\right)=\frac{1}{1-\xi} \log _{p} \frac{1-q^{m}}{1-q}
$$

(3)

$$
G_{p, q, \xi}(x)+\xi^{-1} G_{p, q^{-1}, \xi^{-1}}(1-x)=\frac{1}{1-\xi} \log _{p} q .
$$

In conclusion, I would like to thank Richard Askey and Dennis Stanton for stimulating correspondence and conversations.

\section{REFERENCES}

1. R. Askey, The q-gamma and q-beta functions, Applicable Anal. 8 (1978), 125-141.

2. , Ramanujan's extensions of the gamma and beta functions, Amer. Math. Monthly (to appear).

3. L. Carlitz, q-Bernoulli numbers and polynomials, Duke Math. J. 15 (1948), 987-1000.

4. J. Diamond, The p-adic log gamma function and p-adic Euler constants, Trans. Amer. Math. Soc. 233 (1977), 321-337.

5.

6. B. Ferrero and R. Greenberg, On the behavior of $p$-adic L-functions at $s=0$, Invent. Math. 50 (1978), 91-102.

7. K. Iwasawa, Lectures on p-adic L-functions, Princeton Univ. Press, Princeton, N. J., 1972.

8. F. H. Jackson, On q-definite integrals, Quart. J. Pure and Appl. Math. 41 (1910), 193-203.

9. N. Koblitz, A new proof of certain formulas for p-adic L-functions, Duke Math. J. 46 (1979), 455-468.

10. T. Kubota and H. W. Leopoldt, Eine p-adische Theorie der Zetawerte. I, J. Reine Angew. Math. 214/215 (1965), 328-339.

11. D. Moak, The q-gamma function for $q>1$, Aequationes Math. (to appear).

12. University of Wisconsin Dissertation (to appear).

13. Y. Morita, A p-adic analogue of the $\Gamma$-function, J. Fac. Sc. Univ. Tokyo Sect. IA Math. 22 (1975), 255-266.

Department of Mathematics, University of Washington, Seattle, Washington 98195 